\title{
Babesia spp. in European wild ruminant species: parasite diversity and risk factors for infection
}

\author{
Adam O Michel ${ }^{1}$, Alexander Mathis ${ }^{2}$ and Marie-Pierre Ryser-Degiorgis ${ }^{1 *}$
}

\begin{abstract}
Babesia are tick-borne parasites that are increasingly considered as a threat to animal and public health. We aimed to assess the role of European free-ranging wild ruminants as maintenance mammalian hosts for Babesia species and to determine risk factors for infection. EDTA blood was collected from 222 roe deer (Capreolus c. capreolus), 231 red deer (Cervus e. elaphus), 267 Alpine chamois (Rupicapra r. rupicapra) and 264 Alpine ibex (Capra i. ibex) from all over Switzerland and analysed by PCR with pan-Babesia primers targeting the $18 \mathrm{~S}$ rRNA gene, primers specific for B. capreoli and Babesia sp. EU1, and by sequencing. Babesia species, including B. divergens, B. capreoli, Babesia sp. EU1, Babesia sp. $\mathrm{CH} 1$ and B. motasi, were detected in $10.7 \%$ of all samples. Five individuals were co-infected with two Babesia species. Infection with specific Babesia varied widely between host species. Cervidae were significantly more infected with Babesia spp. than Caprinae. Babesia capreoli and Babesia sp. EU1 were mostly found in roe deer (prevalences $17.1 \%$ and $7.7 \%$, respectively) and B. divergens and Babesia sp. $\mathrm{CH} 1$ only in red deer. Factors significantly associated with infection were low altitude and young age. Identification of Babesia sp. $\mathrm{CH} 1$ in red deer, co-infection with multiple Babesia species and infection of wild Caprinae with B. motasi and Babesia sp. EU1 are novel findings. We propose wild Caprinae as spillover or accidental hosts for Babesia species but wild Cervidae as mammalian reservoir hosts for $B$. capreoli, possibly Babesia sp. EU1 and Babesia sp. $\mathrm{CH1}$, whereas their role regarding $B$. divergens is more elusive.
\end{abstract}

\section{Introduction}

Babesiosis is a tick-borne disease caused by protozoan parasites of the genus Babesia and affecting a wide range of domestic and wild mammalian hosts. Disease signs vary in severity from silent infection to acute circulatory shock with anemia, depending on susceptibility, immunity and age of the host, and on Babesia species and parasite load [1-3]. Worldwide, Babesia species are primarily of veterinary importance [1,4] but human cases mainly reported from North America and Europe have raised the question of whether they may also be emerging human pathogens [5].

In Europe, three Babesia species are of particular interest in ruminants: $B$. divergens, $B$. capreoli and Babesia sp. EU1 (also known as $B$. venatorum [6]). Babesia divergens is the principal agent of babesiosis in cattle [7]. It is capable of infecting gerbils (Meriones unguiculatus), sheep

\footnotetext{
* Correspondence: marie-pierre.ryser@vetsuisse.unibe.ch

${ }^{1}$ Centre for Fish and Wildlife Health, Department of Infectious Diseases and Pathobiology, Vetsuisse Faculty, University of Bern, Bern, Switzerland
} Full list of author information is available at the end of the article
(Ovis aries) and reindeer (Rangifer t. tarandus) [8-10] and was reported in single cases as causative agent of fatal disease in immunosuppressed or splenectomized humans [5]. Babesia capreoli is not known to be pathogenic for humans or livestock $[11,12]$ but is prevalent in freeranging asymptomatic roe deer (Capreolus c. capreolus) $[13,14]$ and occasionally causes disease in wild Caprinae [15]. Babesia sp. EU1 was first identified in 2007 in a human patient from Germany who displayed associated clinical symptoms [16]. Since then, the parasite has been reported in free-ranging roe deer in many European countries including France [17], Germany [18], Slovenia [19], Spain [20] and Poland [21]. Additionally, B. bigemina and $B$. bovis were identified as the cause of babesiosis outbreaks in cattle [22,23]; subclinical infections with $B$. motasi were reported in small domestic ruminants such as goat and sheep [24]; and a new species of Babesia tentatively described as Babesia sp. $\mathrm{CH} 1$ was found in ticks feeding on red deer from Switzerland [25].

Clinical babesiosis in free-ranging wild ruminants appears to be rare. Documented cases concern only Caprinae and were caused by either B. capreoli or B. ovis $[15,26]$. In 
contrast, numerous studies in Europe and abroad have documented the occurrence of silent Babesia spp. infections in free-ranging cervids [3]. However, there has been confusion regarding the identity of the detected Babesia species, particularly in roe deer. While Babesia from roe deer were formerly referred to as $B$. divergens or $B$. divergens-like, recent investigations showed that roe deer are usually infected with $B$. capreoli, which is antigenically and morphologically indistinguishable from $B$. divergens [12]. Differences between the 18S rRNA gene of $B$. divergens and $B$. capreoli have been described at only three positions namely 631, 663 and 1637, with AAC for B. divergens and GTT for B. capreoli [11]; further, differences in the sequences of the internal transcribed spacers 1 and 2 (ITS1, ITS2) have been reported [27]. Relying on nucleotide identities at positions 631 and 663 of the $18 \mathrm{~S}$ rRNA gene, the only confirmed infections with $B$. divergens in free-ranging wild ruminants so far were in red deer from Ireland [28] and more recently in two roe deer from Poland [21].

In Switzerland, occasional outbreaks of babesiosis caused by $B$. divergens have been reported in cattle only [29] but this parasite has been identified in ticks (Ixodes ricinus) collected from both domestic cattle and free-ranging red deer (Cervus e. elaphus) [25]. Between 2005 and 2006, five Alpine chamois succumbed to babesiosis due to B. capreoli $[15,27]$. Subsequent investigations in the two affected Swiss regions tentatively identified roe deer and red deer as potential reservoir for B. capreoli [30]. In that study, however, detected Babesia were not systematically identified to species level, leaving the possibility of prevalence errors. Furthermore, sample size was limited, especially for red deer.

Overall, despite increasing numbers of studies on Babesia spp. in wildlife, gaps of knowledge remain regarding the spectrum of parasite species infecting wild hosts, the potential role of wildlife populations as source of infection for livestock and humans, and the epidemiology of B. capreoli, which causes babesiosis in Alpine chamois. To address these questions, we carried out a country-wide survey in free-ranging indigenous wild ruminants in Switzerland. This country is of particular interest for such a study as it is characterized by various landscapes, climatic patterns and vegetation coverage possibly influencing parasite occurrence (i.e. tick and mammal host occurrence); it also hosts large numbers of four important European wild ruminant species, namely roe deer, red deer, Alpine chamois, and Alpine ibex (Capra ibex ibex). The specific objectives of the study were (1) to document the occurrence and diversity of Babesia species in free-ranging populations of wild ruminants, including the identification of potentially yet undescribed species of Babesia; and (2) to assess risk factors for infection.

\section{Materials and methods}

\section{Study area}

The study area covered the whole territory of Switzerland (41 $285 \mathrm{~km}^{2}$ ), except for the canton of Bern $\left(5659 \mathrm{~km}^{2}\right)$, which did not participate in the sampling campaign due to administrative constraints. Switzerland can be divided into four main bioregions (Jura, Plateau, Alps and South), which differ largely in climate and geographical features [31]. The four wild ruminant species endemic to Switzerland (roe deer, red deer, Alpine chamois and Alpine ibex, with an estimated population size ranging from approximately 15600 for ibex to 113000 for roe deer [32]) show a nonhomogeneous distribution, reflecting the suitability of the landscape as species-specific habitat. The small, introduced populations of Sika deer (Cervus nippon; ca. 250-300 individuals in northern Switzerland) [33] and mufflon (Ovis aries orientalis; ca. 200-300 in south-western Switzerland) [34] were not considered in our study.

\section{Animals and samples}

Blood samples from 984 wild ruminants (222 roe deer, 231 red deer, 267 chamois and 264 ibex) collected from September 2009 to January 2010 in the framework of a cross-sectional study on virus infections [32,35] were used for the present study. Blood was mostly collected from animals hunted, culled or found dead and was sampled by game wardens or hunters using standard sampling kits containing gloves, sterile EDTA tubes and a syringe. We also used samples from five carcasses submitted for post-mortem investigation to the Centre for Fish and Wildlife Health (FIWI) in Bern, Switzerland, and from six live animals captured in the framework of ecological studies. Additionally, we included samples from eight chamois submitted to the FIWI between 2005-2009, confirmed to be infected with B. capreoli and which had died of hemolytic anemia (i.e., five cases previously reported [15] and three more recent cases from 2009). Blood samples from dead animals were collected either directly from the heart or from the body cavities. Samples from live animals were collected by puncturing the jugular vein during chemical immobilization, with the authorizations of competent authorities (see [32]).

Immediately after collection (sampling at the laboratory) or upon receipt (sampling in the field), samples were transferred to $1.5 \mathrm{~mL}$ Eppendorf tubes and frozen at $-20{ }^{\circ} \mathrm{C}$ until further use. Table 1 compiles the demographic and geographic data obtained for each animal by means of a data sheet completed by the submitter.

\section{Laboratory analysis}

DNA was extracted from aliquots of whole EDTA blood using the DNeasy blood \& tissue kit (Qiagen, Hombrechtikon, Switzerland). Analyses were carried out according to the 
Table 1 Demographic data of the animals tested for Babesia infection

\begin{tabular}{ccccc}
\hline Demographic data & \multicolumn{3}{c}{ Species } \\
\cline { 2 - 5 } & Roe deer $(\boldsymbol{n}=\mathbf{2 2 2})$ & Red deer $(\boldsymbol{n}=\mathbf{2 3 1})$ & Alpine chamois $(\boldsymbol{n}=\mathbf{2 6 7})$ & Alpine ibex $(\boldsymbol{n}=\mathbf{2 6 4})$ \\
\hline$<1$-year & 62 & 61 & 7 & 1 \\
A-year & 159 & 169 & 258 & 260 \\
Fge unknown & 1 & 1 & 2 & 3 \\
Female & 117 & 107 & 119 & 129 \\
Male & 105 & 119 & 145 & 134 \\
Sex unknown & 0 & 5 & 3 & 2321.5 \\
Mean altitude & 860 & 1198.4 & 1649.5 & \pm 408.6 \\
St. deviation & \pm 418.1 & \pm 455.7 & \pm 653.1 & $442-2712$ \\
Altitude range & $355-2439$ & $245-2407$ & $431-2909$ & 1 \\
\hline
\end{tabular}

Altitudinal mean and range are both given in meters above sea level (m.a.s.l.).

manufacturer's standard protocol except for the blood quantity and initial incubation step. Due to severe hemolysis or coagulation of some blood samples, sample volume was decreased and incubation period with proteinase $\mathrm{K}$ was extended to increase the final DNA concentration. More specifically, $100 \mu \mathrm{L}$ of EDTA blood were incubated overnight (instead of $200 \mu \mathrm{L}$ incubated for $15 \mathrm{~min}$ ) at $56{ }^{\circ} \mathrm{C}$ with $20 \mu \mathrm{L}$ of proteinase $\mathrm{K}, 100 \mu \mathrm{L}$ phosphate buffered saline and $200 \mu \mathrm{L}$ of buffer AL. In a final step of purification, DNA was eluted in $100 \mu \mathrm{L}$ buffer $\mathrm{AE}$ and stored at $-20{ }^{\circ} \mathrm{C}$ until further use.

DNA was amplified by PCR in $100 \mu \mathrm{L}$ assays prepared as previously described [27] but with $20 \mu \mathrm{L}$ of DNA sample instead of $25 \mu \mathrm{L}$. Table 2 describes primer specifications and PCR cycling conditions. Initially, all samples were screened for Babesia spp. using the pan-Babesia primers BabF/R. Positive samples were then screened for Babesia sp. EU1. Samples positive in the pan-Babesia PCR and either positive or negative for Babesia sp. EU1 were also screened for $B$. capreoli. This was performed using the newly designed specific primers described in Table 2.

DNA samples positive to the pan-Babesia primers but negative to both BabF/EU1R and BabcapF1/R were sequenced. Amplicons obtained from amplification with the pan-Babesia primers were purified using the Qiaquick PCR purification kit (Qiagen) following the manufacturer's instructions. Purified products were sent for sequencing to Synergene Biotech $\mathrm{GmbH}$ (Schlieren, Switzerland). Phylogenetic analyses were conducted using BioNumerics 7 (Applied-Maths NV, Austin, Texas, USA [36]). We constructed a Neighbour-Joining tree with reliability tested using bootstrapping with 1000 pseudoreplicates.

\section{Data management and statistics}

Data coding and management was done in MS Excel and OpenOffice spreadsheets. Statistical analyses were performed with the NCSS 2007 software (Hintze J., 2006; NCSS, Kaysville, Utah, USA [37]). Prevalences were calculated with an assumed test sensitivity and specificity of $100 \%$ (considering the combined results of the pan-Babesia PCR, both specific PCRs and sequencing). Chamois diagnosed with clinical babesiosis were not included in the prevalence calculations because they had not been submitted in the frame of the survey.

We computed a Fisher's exact test (FET) to assess associations between prevalence of infection with different Babesia species and potential risk factors for infection such as host species, sex, age, sampling unit, and cause of death (hunted/culled for humane or population control

Table 2 Primer sequences and PCR conditions used in this study

\begin{tabular}{|c|c|c|c|c|c|c|c|c|}
\hline $\begin{array}{c}\text { Primer } \\
\text { designation }\end{array}$ & Specificity & Locus & Sequence $\left(5^{\prime}-3^{\prime}\right)$ & $\begin{array}{l}\text { Fragment } \\
\text { size }\end{array}$ & $\begin{array}{l}\text { Annealing } \\
\text { temp }\left({ }^{\circ} \mathrm{C}\right)\end{array}$ & $\begin{array}{c}\text { Extension } \\
\text { time (s) }\end{array}$ & No. cycles & Reference \\
\hline BabsppF1 & Babesia spp.* & 18S rRNA gene & GTTTCTGMCCCATCAGCTTGAC & $422-440$ & 61 & 45 & 40 & {$[25]$} \\
\hline BabsppR & & & CAAGACAAAAGTCTGCTTGAAAC & & & & & \\
\hline BabcapF & Babesia capreoli & rRNA locus (ITS2) & AGGAACCACACTITTACTGGTIT & 210 & 62 & 30 & 40 & This study \\
\hline BabcapR & & & CATCCACTTGCYATAGAAATACAA & & & & & \\
\hline BabsppF1 & Babesia sp. EU1 & $18 \mathrm{~S}$ rRNA gene & GTTTCTGMCCCATCAGCTTGAC & 362 & 61 & 45 & 40 & {$[25]$} \\
\hline BabEU1 & & & AGACAAGAGTCAATAACTCGATAAC & & & & & \\
\hline
\end{tabular}

*Bovine Babesia spp.: B. bigemina, B. capreoli, B. canis, B. crassa, B. divergens, B. major, B. motasi, B. odocoilei, B. ovata, Babesia sp. EU1. 
reasons vs. found dead). The Mann-Whitney U test was applied for comparisons of altitudes. Significance level for all tests was set at $p<0.05$. Statistical significance of differences was not assessed for parasite/host combinations with very low prevalence (chamois and ibex for B. capreoli, Babesia sp. EU1 and B. motasi; red deer for $B$. divergens). For the association between sampling unit and prevalence of infection, sampling units with a sample size of less than 10 individuals were not included. For spatial representation and mapping we used QGIS software [38].

\section{Results}

\section{Babesia diversity}

Of 984 tested individuals, 105 (10.7\%) tested positive with pan-Babesia primers, and five different Babesia species could be identified by specific PCRs or sequencing. An overview of the identified Babesia species and number of infected animals is given in Table 3. Babesia capreoli was the most commonly identified species in this study, followed by Babesia sp. EU1, Babesia sp. $\mathrm{CH} 1, B$. divergens and B. motasi. Co-infection with $B$. capreoli and Babesia sp. EU1 was identified in three roe deer and two chamois.

Roe deer had the highest prevalence for Babesia spp. with $B$. capreoli being identified in $17.1 \%$ of the animals. In twelve of these 38 roe deer, PCRs using the B. capreoli specific primers Babcap $\mathrm{F} / \mathrm{R}$ gave negative results, and species identification was achieved by sequencing the pan-Babesia amplicons, revealing $100 \%$ identity to the $B$. capreoli reference sequence from France BAB1220 [GenBank: AY726009]. For verification purposes, two amplicons from samples that were positive with $B$. capreoli and one sample that was positive with Babesia sp. EU1 specific primers were sequenced at the $18 \mathrm{~S}$ rRNA gene (using pan-Babesia primers) revealing $100 \%$ identities with reference sequences (GenBank: AY726009 and GenBank: DQ312434, respectively). One roe deer amplicon could not be characterized due to poor sequence quality.
In red deer, the amplicons of six samples were identified as $B$. divergens of bovine origin [GenBank: AY046576], and 11 as Babesia sp. CH1 [Genbank: DQ312432]. Amplicons from 23 red deer were only identifiable to genus level due to insufficient sequence quality (weak signal strength, short segment reads or unclear nucleotide designation).

Babesia prevalences were low in Alpine chamois and Alpine ibex, and B. motasi [GenBank: AY260180] was identified in four animals.

All sequences of $B$. divergens analysed in this study form a clade separated from the one made of B. capreoli sequences, supported by $65 \%$ bootstrapping (Figure 1). Sequences of Babesia sp. EU1 cluster with reference sequences to which they are identical, as do B. motasi sequences. Babesia sp. $\mathrm{CH} 1$ from red deer is clearly separated from B. odocoilei (supported by $89 \%$ bootstrapping), which is the closest known Babesia species (Figure 1).

\section{Risk factors for infection \\ Host species}

Prevalence of infection with Babesia spp. did not significantly differ within Cervidae, i.e. between roe deer and red deer $(p=0.103)$, or within Caprinae, i.e. between ibex and chamois $(p=0.382)$. In contrast, there was a significant difference between Cervidae and Caprinae $(p<0.0001)$

Prevalence of infection with specific Babesia varied widely among host species (Table 3 ). Babesia capreoli was detected more often in roe deer $(17.1 \%)$ than chamois $(n=2,0.8 \%, p<0.0001)$ and in none of the tested red deer and ibex $(p<0.0001$ and $p<0.0001$, respectively). Similarily, Babesia sp. EU1 was found more frequently in roe deer $(7.7 \%)$ than chamois $(n=7,2.6 \%, p=0.01)$ and was found in only one ibex $(0.4 \%, p<0.0001)$ and none of the red deer $(p<0.0001)$. Co-infections with $B$. capreoli and Babesia sp. EU1 were detected in three roe deer and two chamois. Babesia divergens and Babesia sp. $\mathrm{CH} 1$ were only detected in red deer, and B. motasi was identified only in chamois and ibex.

Table 3 Prevalences of the different Babesia species identified in four species of wild ruminants

\begin{tabular}{|c|c|c|c|c|c|c|c|c|}
\hline & \multicolumn{2}{|c|}{ Roe deer $(n=222)$} & \multicolumn{2}{|c|}{ Red deer $(n=231)$} & \multicolumn{2}{|c|}{ Alpine chamois $(n=267)$} & \multicolumn{2}{|c|}{ Alpine ibex $(n=264)$} \\
\hline & No. infected & $\begin{array}{c}\text { Prevalence } \\
(95 \% \mathrm{CI})\end{array}$ & No. infected & $\begin{array}{c}\text { Prevalence } \\
(95 \% \mathrm{Cl})\end{array}$ & No. infected & $\begin{array}{c}\text { Prevalence } \\
(95 \% \mathrm{Cl})\end{array}$ & No. infected & $\begin{array}{c}\text { Prevalence } \\
(95 \% \mathrm{Cl})\end{array}$ \\
\hline Babesia spp. & 53 & $23.9 \%(18.4-30.0)$ & 40 & $17.3 \%(12.7-22.8)$ & 8 & $3.0 \%(1.3-5.8)$ & 4 & $1.5 \%(0.4-3.8)$ \\
\hline B. capreoli & 38 & $17.1 \%(12.4-22.7)$ & & & 2 & $0.8 \%(0.1-2.7)$ & & \\
\hline B. divergens & & & 6 & $2.6 \%(1.0-5.6)$ & & & & \\
\hline Babesia sp. EU1 & 17 & $7.7 \%(4.5-12.0)$ & & & 7 & $2.6 \%(1.1-5.3)$ & 1 & $0.38 \%(0.01-2.1)$ \\
\hline Babesia sp. $\mathrm{CH} 1$ & & & 11 & $4.8 \%(2.4-8.4)$ & & & & \\
\hline B. motasi & & & & & 1 & $0.4 \%(0.01-2.07)$ & 3 & $1.1 \%(0.2-3.3)$ \\
\hline
\end{tabular}

Prevalence is calculated as the number of infected individuals over the total number of individuals tested within the same wild ruminant species. The 95\% confidence interval $(95 \% \mathrm{Cl})$ is given in brackets beside the prevalence. Prevalence for Babesia spp. includes all positive individuals with the pan-Babesia PCR. Prevalences for the different Babesia species were calculated based on results of the specific PCRs or sequencing (incomplete sequences from one roe deer and 23 red deer excluded). Co-infected individuals with B. capreoli/Babesia sp. EU1 (three roe deer and two chamois) were considered once for each Babesia species. 


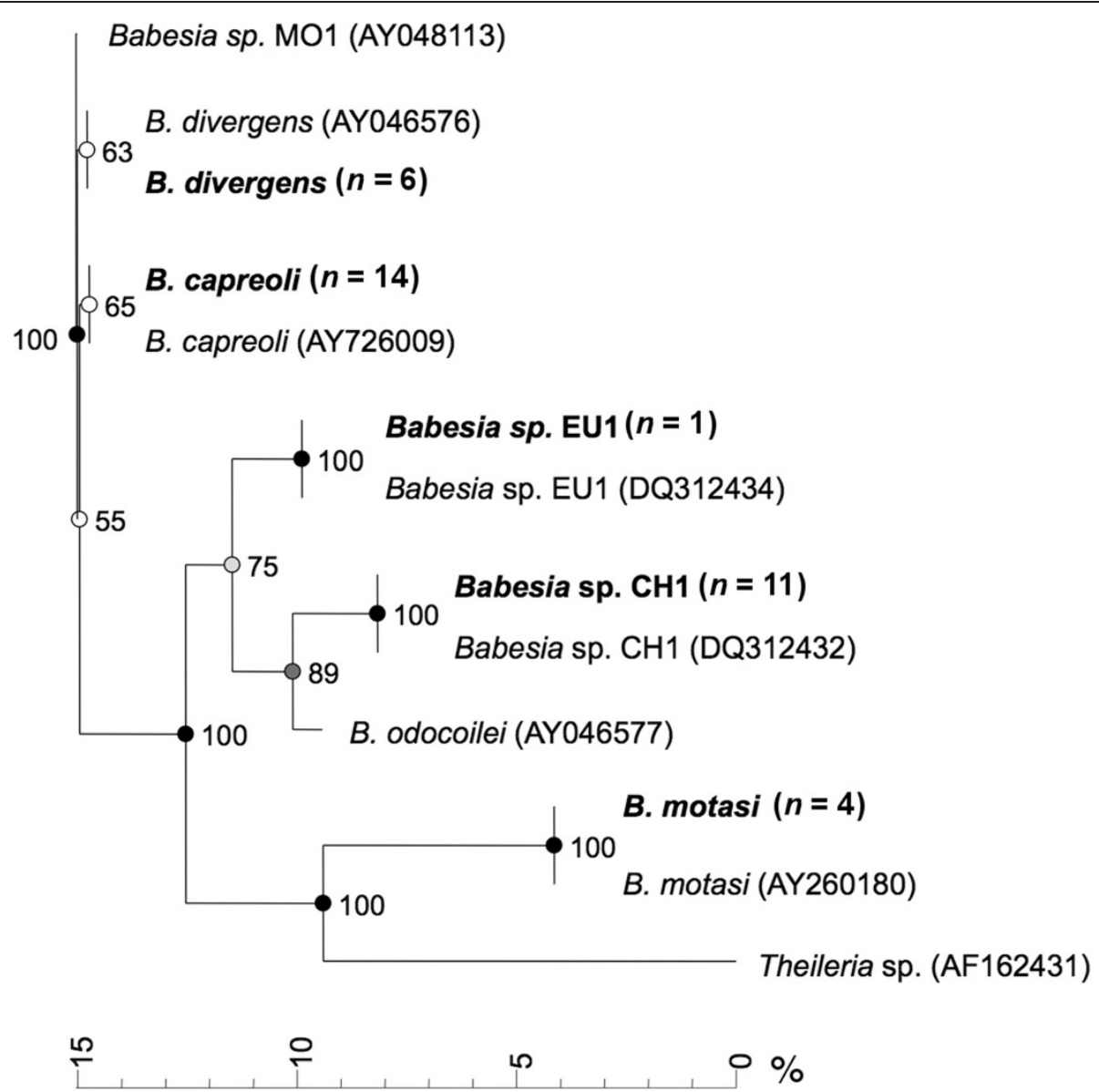

Figure 1 Neighborhood joining tree of partial Babesia 18S rRNA gene sequences. Sequences from wild ruminants from the present study are highlighted in bold (number of identical sequences in brackets). Selected reference piroplasm sequences from GenBank (accession numbers in brackets) are also shown. Bootstrap values indicated at each node base are on $\mathrm{N}=1000$ replicates. Bar $=$ percentage of difference between sequences.

Sex

We found no relationship between sex and Babesia infection, both when Babesia spp. and all species of ruminants were considered together $(P=0.406)$ and when Babesia species and host species were looked at independently.

\section{Age}

There was a significant association between young age and infection with Babesia spp. in roe deer. Twentythree of $62(37.1 \%)$ roe deer kids (<1-year old) were infected with Babesia spp. as opposed to 29 of 159 (18.2\%) roe deer that were 1 -year or older $(p=0.00451)$. This was also observed when different Babesia species were considered separately (B. capreoli, $p=0.0148$; Babesia sp. EU1, $p=0.0246$ ). Furthermore, four out of the five individuals showing concurrent infections with both B. capreoli and Babesia sp. EU1 were less than 1 year of age.

\section{Altitude}

Regardless of the infection status, mean altitudes of sampling sites significantly differed among wild ruminant species $(p<0.0001)$. Cervidae were found at significantly lower altitudes $(\bar{x}=1031.5$ m.a.s.l., $S D=466.7)$ than Caprinae $(\mu=1972.5$ m.a.s.l., $\mathrm{SD}=645.4 ; p<0.0001)$. All host species combined, individuals positive for Babesia spp. were found at significantly lower altitudes $(\bar{x}=893.8$, $\mathrm{SD}=485.6)$ than individuals that were not $(\overline{\mathrm{x}}=1616.4$, $\mathrm{SD}=725.5 ; p<0.0001)$. This altitudinal difference was also observed when each host species was analysed independently. Roe deer positive for B. capreoli were sampled at significantly lower altitudes $(\mu=682.9$ m.a.s.l., $\mathrm{SD}=$ 258.4) than negative individuals $(\mu=897.3$ m.a.s.l., $\mathrm{SD}=$ 434.6; $p=0.0036$ ). Similarly, the mean altitude of red deer positive for Babesia sp. $\mathrm{CH} 1 \quad(\mu=858.7$ m.a.s.l., $\mathrm{SD}=$ 327.2) was significantly lower than that of negative individuals $(\mu=1212.6$ m.a.s.l., $\mathrm{SD}=452.7, p=0.0062)$. In contrast, this was not observed among roe deer infected with 
Babesia sp. EU1 (positive: $\mu=720.29$ m.a.s.l., SD = 230.55; negative: $\mu=872.7$ m.a.s.l., $\mathrm{SD}=427.42 ; p=0.218$ ).

\section{Geographic region}

No differences of prevalence between the different sampling units were observed, neither for B. capreoli $(p=0.132$ to $p=1.000)$ and Babesia sp. EU1 ( $p=0.175$ to $p=1.000)$ among roe deer, nor for $B$. divergens $(p=0.412$ to $p=$ $1.000)$ and Babesia sp. $\mathrm{CH} 1(p=0.125$ to $p=0.569)$ among red deer (Figures 2 and 3). Only B. motasi was confined to the South-West sampling unit (Figures 2 and 3).

\section{Cause of death}

Babesia sp. EU1 infection was significantly more common in roe deer found dead $(17.1 \%, p=0.039)$ than in those hunted (6.0\%). None of the three roe deer and two chamois co-infected with Babesia sp. EU1 and B. capreoli were found dead. An association between infection and cause of death was not observed for any other host/ parasite species combination.

\section{Discussion}

Our study aimed at determining the occurrence and diversity of Babesia species in Swiss wild ruminants and at assessing risk factors for infection, in order to better understand the role of roe deer, red deer, Alpine chamois and Alpine ibex in the epidemiology of babesiosis of wildlife, livestock and humans.

\section{Babesia divergens}

We report for the first time the identification of $B$. divergens in red deer in Switzerland. Very few studies have focused on the identification of $B$. divergens in red deer in Europe, and in the only other study reporting $B$. divergens in red deer in continental Europe, no attempt was made to confirm the findings by sequencing [14]. Our estimated prevalence for $B$. divergens in red deer $(2.6 \%)$ is much lower than reported in Ireland and Slovenia $(29.0 \%$ and $16.7 \%$, respectively $[14,28])$. However, no Babesia species identification could be achieved for as many as 23 of the 40 infected red deer due to poor sequence data. As this

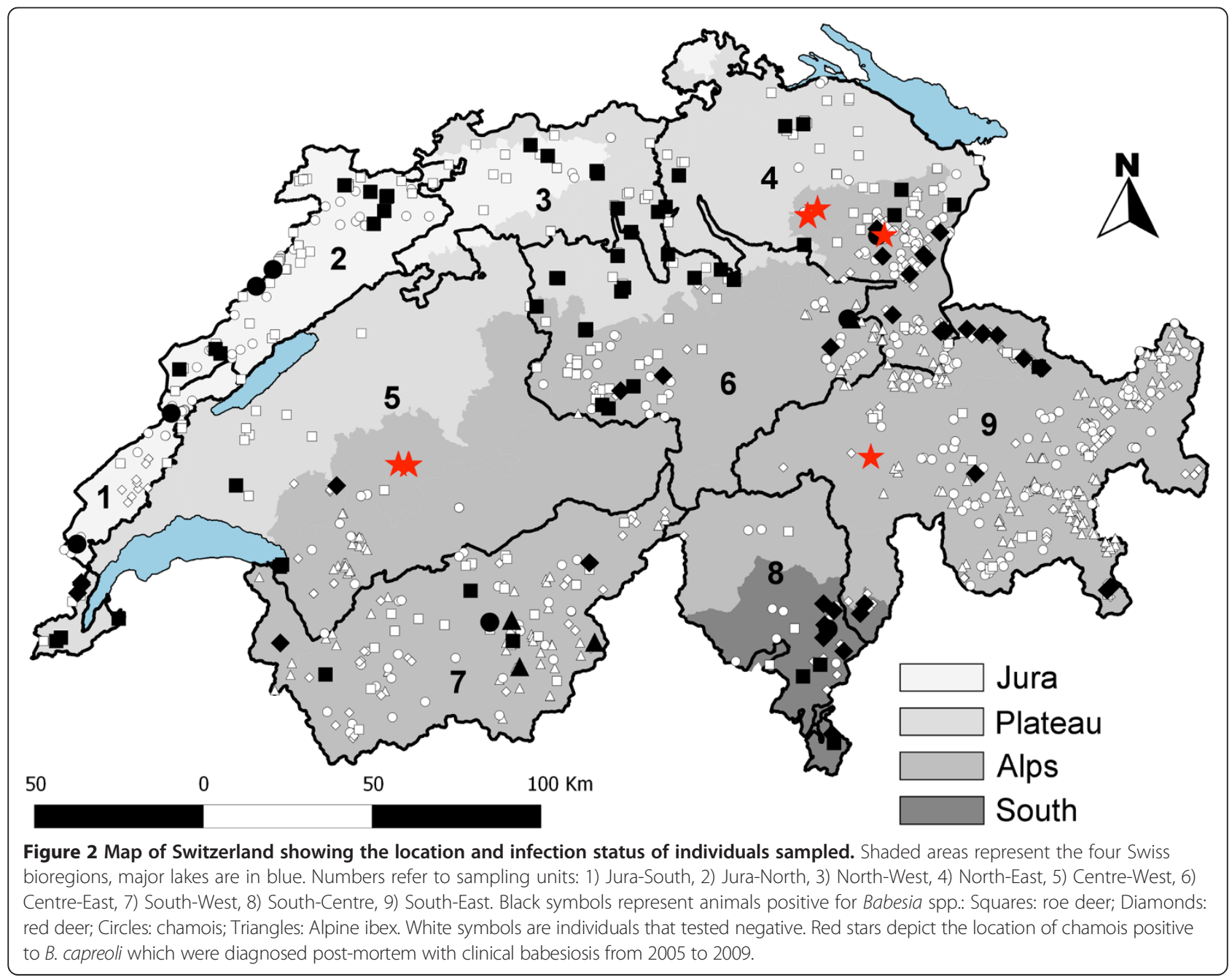




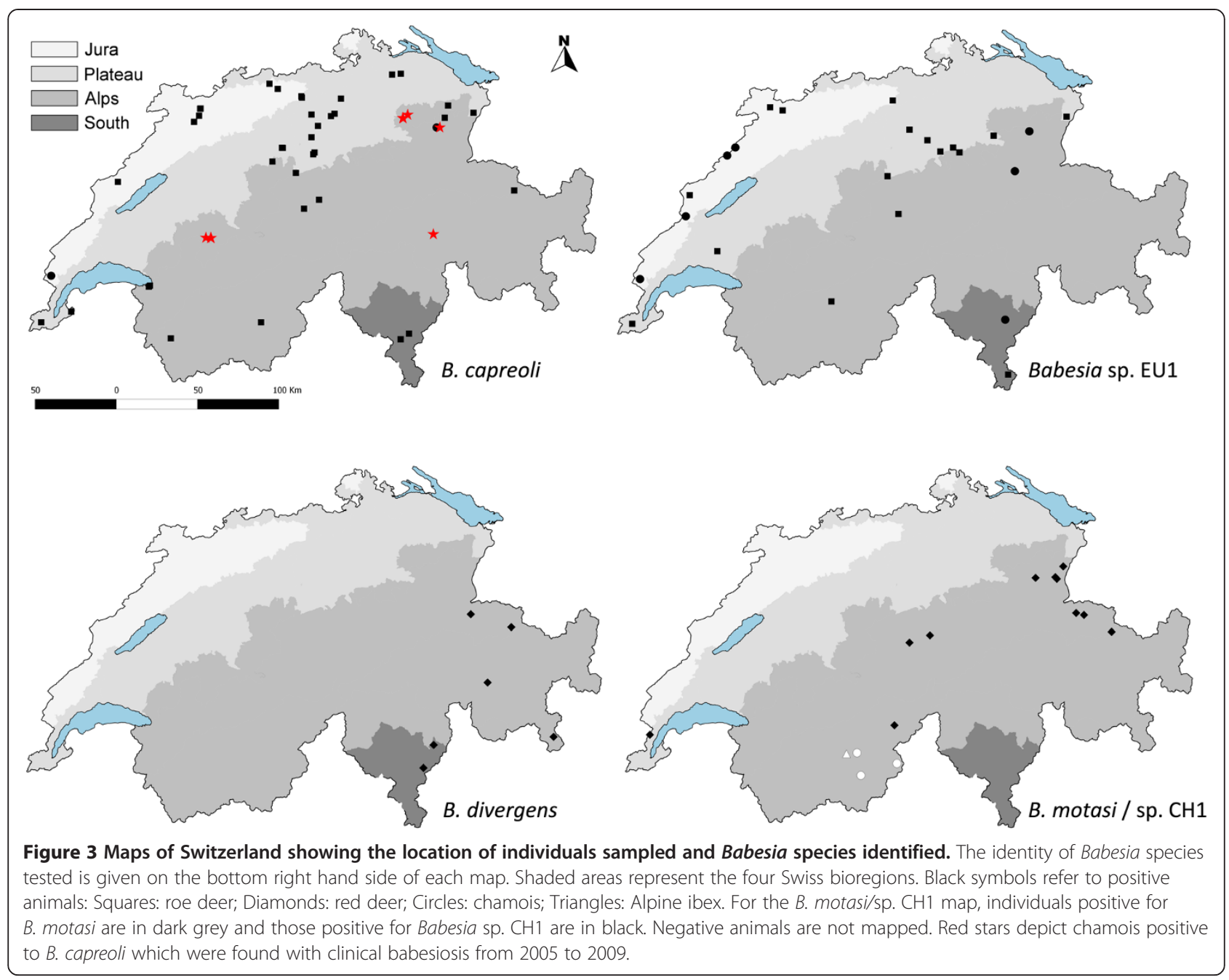

occurred only in one roe deer and none of the Caprinae, it seems unlikely that varying sample quality would account for these differences. Additionally, it was shown that serum obtained with the same red deer blood samples (simultaneously collected in different tubes and used for another study [32]) were not more haemolytic than those from other wild ruminant species. Interestingly, Zintl et al. [28] also reported poor sequence quality in many red deer samples, and the reason for this apparently red deerspecific phenomenon remains unclear.

Because of a relatively large proportion of unidentified amplicons, our Babesia-specific prevalences in red deer are underestimated. If all of these 23 samples were $B$. divergens, the prevalence (12.6\%) would be in a comparative range as in the two other studies. Alternatively, a lower prevalence in the red deer of our study could be explained by the fact that the animals were sampled at higher altitudes than in Slovenia (300-500 m; T. Avsic, personal communication) and Ireland (highest peak at sampling sites: $842 \mathrm{~m}$ [39]), suggesting a lower exposure to ticks.
Roe deer have been extensively studied and proposed as a potential host for $B$. divergens but to date, only Welc-Faleciak et al. have identified two roe deer infected with $B$. divergens. However, these two isolates had two unique polymorphic sites in the highly conserved $18 \mathrm{~S}$ rRNA gene, hence casting doubt as to their proper identity as $B$. divergens [21]. Our present results suggest that $B$. divergens does not occur in roe deer and converge with the observation of Malandrin et al. [11], who concluded from experimental in vitro erythrocyte infection studies that roe deer are not favourable hosts for infection with $B$. divergens. Taken together, these findings suggest that in Switzerland, cervids may not play an important role as primary or mammalian maintenance hosts for $B$. divergens, but an estimate of prevalence in domestic cattle and data on red deer from areas located at lower altitude would be necessary to better address this point.

\section{Babesia capreoli}

Of the 38 roe deer infected with $B$. capreoli in our study, 24 were positive by PCR with primers that target a 
region of the rDNA ITS2 domain known to discriminate between B. capreoli and B. divergens [27]. To our knowledge, this is the first time that primers have been designed and successfully used to identify samples positive for $B$. capreoli. However, a smaller portion of these samples $(n=14)$ were initially negative with $B$. capreoli primers but matched with $100 \%$ identity to B. capreoli of roe deer origin [GenBank: AY726009] after sequencing the pan-Babesia amplicon. The reason for the apparent lack of sensitivity of the primers is unclear. One putative factor is the modification of the annealing temperature, which had to be increased from $60{ }^{\circ} \mathrm{C}$ to $62{ }^{\circ} \mathrm{C}$ because of cross-reactivity with $B$. divergens control DNA (not shown). Furthermore, given the limited knowledge about the amplified region of the rDNA ITS2, there could be intra-specific variation within the primer binding sites that could account for this difference.

The relatively high prevalence of $B$. capreoli in roe deer (17.1\%), which does not significantly differ from previous studies from Switzerland (26.1\%) and Poland (11.9\%) [21,30] suggests that roe deer are mammalian maintenance hosts for B. capreoli. Red deer however, do not seem to be susceptible to infection.

In the current study, B. capreoli was detected in samples from two apparently healthy chamois. Together with earlier data $[15,30]$, our finding of a very low prevalence of B. capreoli in Alpine chamois suggests that they are spillover, accidental hosts which mostly succumb to disease upon infection. Indeed, of a total of 317 chamois without reported disease signs, only four were PCR positive $(1.3 \%)$ while all eight chamois with fatal hemolytic anemia and a marked parasitemia were infected ([15,30]; this study). Furthermore, while diseased animals were thoroughly examined, absence of disease and parasite identification were not definitely confirmed in subclinical infections. Nevertheless, the detection of a few chamois that apparently do not develop disease may be related to host factors such as innate resistance or protective immunity due to exposure early in life $[40,41]$, as well as parasite-specific factors such as differences in the pathogenic potential of various strains [42]. In a former study, B. capreoli sequences identified in Alpine chamois that had died of clinical babesiosis [GenBank: EU182596] were identical to those of B. capreoli from roe deer, when near full-length $18 \mathrm{~S}$ rRNA gene sequences were compared to each other.

\section{Babesia sp. EU1}

Babesia sp. EU1 was identified in roe deer, Alpine chamois and Alpine ibex. Previous studies have shown that this Babesia species is common in roe deer [14,17], and given the findings of our study we suggest that roe deer is a mammalian maintenance host for this parasite. Furthermore, to our knowledge, we report for the first time the occurrence of Babesia sp. EU1 in Caprinae and document their status as spillover hosts for this parasite. So far, Babesia sp. EU1 has never been isolated from a red deer and we provide further evidence that red deer may not be susceptible to infection. Babesia spp. are mostly described in the literature as causing infection in only one host. However, Babesia sp. EU1, as we document, is able to infect at least three hosts, namely roe deer, Alpine chamois and Alpine ibex. Although our data set does not exclude the possibility of clinical disease due to Babesia sp. EU1 in these hosts, there is little evidence to support that contention. However, it is interesting that roe deer found dead were significantly more frequently infected with Babesia sp. EU1 than hunted roe deer, raising the possibility that infection with Babesia sp. EU1 may have contributed to mortality.

Concurrent infections with $B$. capreoli and Babesia sp. EU1 Concurrent infections of mammalian hosts with multiple Babesia species have not been reported to date. However, co-infections of mammalian hosts with tick-borne pathogens of different genera are known to occur, including simultaneous infection with Babesia and Theileria (reported in cattle) [43] and co-infection with Babesia and Borrelia burgdorferi (observed in humans) [44]. Similarily, infection of ticks with multiple pathogens has been reported [45-47]. The lack of identification of co-infections with two or more Babesia species in mammalian hosts may predominantly result from the applied methods of genetic analysis, which only identify single Babesia species from samples. Consequently, multiplex (real-time) PCRs or reverse line blot hybridization should be used to confidently exclude co-infections. Using our PCR-based approach, co-infection status with two Babesia species became apparent in five animals. Interestingly, the two apparently healthy Alpine chamois infected with B. capreoli were also infected with Babesia sp. EU1, and none of the three roe deer with co-infection had been found dead, raising the possibility that co-infection may dampen the pathogenic effect of either Babesia species. Indeed, experimental co-infection with $B$. divergens and Anaplasma phagocytophila in cattle resulted in markedly reduced hematological abnormalities when compared with animals infected with either pathogen [48]. However, another study suggested that co-infection with two hemoparasites of low virulence can have additive effects and lead to disease, while infection with either one would remain subclinical [43].

\section{Babesia motasi}

Babesia motasi was identified in three Alpine ibex and one chamois, all originating from the sampling unit South-West. It has never been identified before in wildlife, but the European strain of B. motasi - unlike the highly 
virulent Turkish strain - is a parasite found at low prevalence in sheep and goats in Europe and it does not cause illness [24,49-51]. Haemaphysialis punctata is the known vector of B. motasi [52] and interestingly in Switzerland this tick species only occurs in the unit South-West [53]. Our findings suggest that Alpine chamois and ibex are hosts of $H$. punctata in southern Switzerland and show that B. motasi is able to infect wild Caprinae. The low prevalence at which the parasite is present in these species suggests they are occasional spillover hosts. Given the apparently low pathogenic nature of the parasite, it is expected to pose little risk for domestic or wild ruminant health.

\section{Babesia sp. $\mathrm{CH} 1$}

Babesia sp. $\mathrm{CH} 1$ was first discovered in I. ricinus ticks feeding on red deer from Switzerland [25] and we show for the first time in this study that the parasite is able to infect red deer. Because the animals sampled were apparently healthy, hunted individuals, there is no indication that Babesia sp. $\mathrm{CH} 1$ is pathogenic to red deer. Mortality has not been reported in other ruminant species either. Phylogenetically, this parasite is most closely related to B. odocoilei, the Babesia species of the NorthAmerican white-tailed deer, transmitted by $I$. scapularis [54]. The wide spectrum of sequences of this and other similar but not identical $B$. odocoilei-like parasites that have been identified in previous studies $[25,28,55]$ suggests a parasite whose genome may have radiated from a single origin and is well established within the European red deer populations. Given that no other host from our study was positive for this parasite, we hypothesize that red deer is the only susceptible host for this species of Babesia among Alpine free-ranging wild ruminants.

\section{Risk factors for infection}

Besides the obvious host-predilection of Babesia species identified in this study, age and altitude were found to account for differences in prevalence. Ibex and chamois (Caprinae, prevalence of 2.3\%) are less likely than Cervidae (21\%) to encounter ticks given the altitude at which they are usually found; it is well reported that tick density decreases with increasing altitude [56,57]. However, our results only partially support the contention that positive animals are more likely to be found at lower altitudes than negative individuals. While B. capreoli in roe deer (this study and [30]) and Babesia sp. CH1 in red deer are associated with lower altitudinal ranges, it does not seem to be the case for infection with Babesia sp. EU1 in roe deer. Nevertheless, this may be due to the occurrence of the parasite in low-lying geographical regions in which the small altitudinal range of the host does not allow any distinction between the location of positive and negative individuals.
Our results suggest that roe deer kids are more often infected with B. capreoli or Babesia sp. EU1 than are adults. In cattle, it has been shown that calves show few, if any clinical signs of disease upon infection with $B$. bovis and may become persistently infected [40]. In Przewalski horses (Equus ferus przewalskii), individuals which are not challenged with equine piroplasms at an early age are unable to cope with an infection in their adult years [41]. These data indicate that exposure early in life determines the outcome of an infection at adult age. Thus, first exposure of roe deer to B. capreoli or Babesia sp. EU1 at an early age may result in a detectable parasitemia, which may be later reduced to a non-detectable level or cleared by the immune reaction, and lead to a long-lasting protective immunity preventing re-infection.

Except for B. motasi, which is confined to the South-West sampling unit, our results do not suggest a particular geographical region as a risk factor for infection. The North-East bioregion, in which the first chamois that died of babesiosis were previously found, did not show a higher prevalence of $B$. capreoli. Although this may be due to a low sample size at local level, it underlines the importance of considering other aspects not measured in our study, such as vector and host occurrence.

\section{Conclusions}

In this study, we have documented the occurrence and diversity of Babesia species in a large number of freeranging ruminants in Switzerland, reporting both previously catalogued and newly discovered parasites in wild ruminants. We show that species of European wild ruminants can be hosts for a range of Babesia species; additionally, one individual can be simultaneously infected with more than one species of Babesia. Conversely, we also show that certain species of Babesia are not specific to one host species.

Furthermore, we propose that cervids are mammalian reservoir hosts for B. capreoli (roe deer) and possibly also for Babesia sp. EU1 (roe deer) and Babesia sp. CH1 (red deer) while their epidemiological role regarding $B$. divergens is more difficult to assess. In contrast, caprids seem to be only spillover or accidental hosts for all Babesia species recorded in our study. The occurrence of apparently healthy free-ranging ruminants infected with $B$. divergens or Babesia sp. EU1 is an important finding, given the pathogenic potential of these parasites for domestic livestock and/or humans and the wide distribution of their tick vector I. ricinus [17].

Finally, the presence of co-infected individuals as well as the higher prevalence of B. capreoli and Babesia sp. EU1 in juveniles than in adults are interesting from an immunological point of view. First, it converges with former observations that infection early in life does not lead to clinical disease. Second, it questions whether 
infection with a certain species of Babesia may provide cross-protection against the pathogenic effects of a subsequent infection with another Babesia species.

\section{Competing interests}

The authors declare that they have no competing interests.

\section{Authors' contributions}

AOM contributed to the study design and sample collection, performed the molecular and data analyses and drafted the manuscript. AM contributed to the study design and supervised molecular analyses. MPR designed the study, supervised the sample collection and data analysis and drafted the manuscript. All authors critically read and approved the final manuscript. This manuscript is part of the inaugural dissertation of AOM.

\section{Acknowledgements}

Authors thank all game wardens, hunters and cantonal hunting offices for their assistance in sample collection. Many thanks also to Fabien Mavrot, Nelson Marreros, Helena Pia Greter, Natacha Wu and Manuela Weber for processing samples, and to Jeannine Hauri for excellent technical assistance. We would also like to extend a special thanks to Francesco Origgi for his ideas and suggestions as well as to Cord Drogemüller for kindly granting us access to his facilities. Sample collection occurred in the frame of a project supported by the Swiss Federal Veterinary Office (Reference 1.10.07) and the Federal Office of Environment (1301-1459); sample analysis was made possible thanks to a grant from the Federal Office of the Environment (Credit no. A2310.0127).

\section{Author details}

'Centre for Fish and Wildlife Health, Department of Infectious Diseases and Pathobiology, Vetsuisse Faculty, University of Bern, Bern, Switzerland. ${ }^{2}$ Swiss National Centre for Vector Entomology, Institute of Parasitology, Vetsuisse Faculty, University of Zurich, Zurich, Switzerland.

Received: 9 January 2014 Accepted: 27 May 2014

Published: 13 June 2014

\section{References}

1. Zintl A, Mulcahy G, Skerrett HE, Taylor SM, Gray JS: Babesia divergens, a bovine blood parasite of veterinary and zoonotic importance. Clin Microbiol Rev 2003, 16:622-636.

2. Homer MJ, Aguilar-Delfin I, Telford SR 3rd, Krause PJ, Persing DH: Babesiosis. Clin Microbiol Rev 2000, 13:451-469.

3. Penzhorn BL: Babesiosis of wild carnivores and ungulates. Vet Parasitol 2006, 138:11-21.

4. L'Hostis M, Chauvin A, Valentin A, Marchand A, Gorenflot A: Large scale survey of bovine babesiosis due to Babesia divergens in France. Vet Rec 1995, 136:36-38.

5. Hildebrandt A, Gray JS, Hunfeld K-P: Human babesiosis in Europe: what clinicians need to know. Infection 2013, 41:1057-1072.

6. Herwaldt BL, Cacciò S, Gherlinzoni F, Aspöck H, Slemenda SB, Piccaluga P, Martinelli G, Edelhofer R, Hollenstein U, Poletti G, Pampiglione S, Löschenberger K, Tura S, Pieniazek NJ: Molecular characterization of a non-Babesia divergens organism causing zoonotic babesiosis in Europe. Emerg Infect Dis 2003, 9:942-948.

7. L'Hostis M, Chauvin A: Babesia divergens in France: descriptive and analytical epidemiology. Parassitologia 1999, 41(Suppl 1):59-62.

8. Chauvin A, Valentin A, Malandrin L, L'Hostis M: Sheep as a new experimental host for Babesia divergens. Vet Res 2002, 33:429-433.

9. Langton C, Gray JS, Waters PF, Holman PJ: Naturally acquired babesiosis in a reindeer (Rangifer tarandus tarandus) herd in Great Britain. Parasitol Res 2003, 89:194-198.

10. Lewis $\mathrm{D}$, Williams $\mathrm{H}$ : Infection of the Mongolian gerbil with the cattle piroplasm Babesia divergens. Nature 1979, 278:170-171.

11. Malandrin L, Jouglin M, Sun Y, Brisseau N, Chauvin A: Redescription of Babesia capreoli (Enigk and Friedhoff, 1962) from roe deer (Capreolus capreolus): isolation, cultivation, host specificity, molecular characterisation and differentiation from Babesia divergens. Int J Parasitol 2010, 40:277-284.
12. Gray JS, Murphy TM, Taylor SM, Blewett DA, Harrington R: Comparative morphological and cross transmission studies with bovine and deer babesias in Ireland. Prev Vet Med 1990, 9:185-193.

13. Bastian S, Jouglin M, Brisseau N, Malandrin L, Klegou G, L'Hostis M, Chauvin A: Antibody prevalence and molecular identification of Babesia spp. in roe deer in France. J Wild Dis 2012, 48:416-424.

14. Duh D, Petrovec M, Bidovec A, Avsic-Zupanc T: Cervids as Babesiae hosts, Slovenia. Emerg Infect Dis 2005, 11:1121-1123.

15. Hoby S, Robert N, Mathis A, Schmid N, Meli ML, Hofmann-Lehmann R, Lutz H, Deplazes P, Ryser-Degiorgis M-P: Babesiosis in free-ranging chamois (Rupicapra r. rupicapra) from Switzerland. Vet Parasitol 2007, 148:341-345.

16. Häselbarth K, Tenter AM, Brade V, Krieger G, Hunfeld K-P: First case of human babesiosis in Germany - Clinical presentation and molecular characterisation of the pathogen. Int J Med Microbiol 2007, 297:197-204.

17. Bonnet $\mathrm{S}$, Jouglin $\mathrm{M}$, L'Hostis $\mathrm{M}$, Chauvin A: Babesia sp. EU1 from roe deer and transmission within Ixodes ricinus. Emerg Infect Dis 2007, 13:1208-1210.

18. Overzier E, Pfister K, Herb I, Mahling M, Böck G Jr, Silaghi C: Detection of tick-borne pathogens in roe deer (Capreolus capreolus), in questing ticks (Ixodes ricinus), and in ticks infesting roe deer in southern Germany. Ticks Tick Borne Dis 2013, 4:320-328.

19. Duh D, Petrovec M, Avsic-Zupanc T: Molecular characterization of human pathogen Babesia EU1 in Ixodes ricinus ticks from Slovenia. J Parasitol 2005, 91:463-465.

20. García-Sanmartín J, Aurtenetxe O, Barral M, Marco I, Lavin S, García-Pérez AL, Hurtado A: Molecular detection and characterization of piroplasms infecting cervids and chamois in Northern Spain. Parasitology 2007, 134:391-398.

21. Welc-Falęciak R, Werszko J, Cydzik K, Bajer A, Michalik J, Behnke JM: Co-infection and genetic diversity of tick-borne pathogens in roe deer from Poland. Vector Borne Zoonotic Dis 2013, 13:277-288.

22. Hilpertshauser H, Deplazes P, Meli ML, Hofmann-Lehmann R, Lutz H, Mathis A: Genotyping of Babesia bigemina from cattle from a non-endemic area (Switzerland). Vet Parasitol 2007, 145:59-64.

23. Savini G, Conte A, Semproni G, Scaramozzino P: Tick-borne diseases in ruminants of Central and Southern Italy: epidemiology and case reports. Parassitologia 1999, 41(Suppl 1):95-100.

24. Friedhoff KT: Tick-borne diseases of sheep and goats caused by Babesia, Theileria or Anaplasma spp. Parassitologia 1997, 39:99-109.

25. Hilpertshauser H, Deplazes P, Schnyder M, Gern L, Mathis A: Babesia spp. identified by PCR in ticks collected from domestic and wild ruminants in Southern Switzerland. Appl Environ Microbiol 2006, 72:6503-6507.

26. Marco I, Velarde R, Castellà J, Ferrer D, Lavín S: Presumptive Babesia ovis infection in a Spanish ibex (Capra pyrenaica). Vet Parasitol 2000, 87:217-221.

27. Schmid N, Deplazes P, Hoby S, Ryser-Degiorgis M-P, Edelhofer R, Mathis A: Babesia divergens-like organisms from free-ranging chamois (Rupicapra $r$. rupicapra) and roe deer (Capreolus c. capreolus) are distinct from B. divergens of cattle origin - an epidemiological and molecular genetic investigation. Vet Parasitol 2008, 154:14-20.

28. Zintl A, Finnerty EJ, Murphy TM, de Waal T, Gray JS: Babesias of red deer (Cervus elaphus) in Ireland. Vet Res 2011, 42:7.

29. Gern L, Brossard M, Aeschlimann A, Broquet CA, Quenet G, Stucki JP, Ackermann J: Bovine piroplasmosis in the Clos-du-Doubs (Jura, Switzerland): preliminary observations. Schweiz Arch Tierheilkd 1982, 124:549-556.

30. Hoby S, Mathis A, Doherr MG, Robert N, Ryser-Degiorgis M-P: Babesia capreoli infections in Alpine chamois (Rupicapra r. rupicapra), roe deer (Capreolus c. capreolus) and red deer (Cervus elaphus) from Switzerland. $J$ Wildl Dis 2009, 45:748-763.

31. Die biogeographischen Regionen der Schweiz - Bundesamt für Umwelt BAFU. [http://www.bafu.admin.ch/publikationen/publikation/00207/index. html? lang=de]

32. Casaubon J, Chaignat V, Vogt H-R, Michel AO, Thür B, Ryser-Degiorgis M-P: Survey of bluetongue virus infection in free-ranging wild ruminants in Switzerland. BMC Vet Res 2013, 9:166.

33. Sikahirsch - Wildtier Schweiz. [http://www.bafu.admin.ch/tiere/09262/ 09387/index.html?lang=fr]

34. Mouflon - Office fédéral de l'environnement OFEV. [http://www.bafu. admin.ch/tiere/09262/09428/index.html?lang=fr] 
35. Casaubon J, Vogt H-R, Stalder H, Hug C, Ryser-Degiorgis M-P: Bovine viral diarrhea virus in free-ranging wild ruminants in Switzerland: low prevalence of infection despite regular interactions with domestic livestock. BMC Vet Res 2012, 8:204

36. BioNumerics Seven | Applied Maths. [http://www.applied-maths.com/ bionumerics]

37. NCSS | Statistical Software | Data Analysis | Graphics Software | NCSS. com. [http://www.ncss.com/software/ncss/]

38. QGIS Development Team: QGIS Geographic Information System. Open Source Geospatial Foundation Project. QGIS Development Team; 2013.

39. EUNIS - Site factsheet for Cloghernagore Bog and Glenveagh National Park. [http://eunis.eea.europa.eu/sites/IE0002047]

40. Zintl A, Gray JS, Skerrett HE, Mulcahy G: Possible mechanisms underlying age-related resistance to bovine babesiosis. Parasite Immunol 2005, 27:115-120.

41. Rüegg SR, Torgerson PR, Doherr MG, Deplazes P, Böse R, Robert N, Walzer C: Equine piroplasmoses at the reintroduction site of the Przewalski's horse (Equus ferus przewalskii) in Mongolia. J Wildl Dis 2006, 42:518-526.

42. Lau AO, Kalyanaraman A, Echaide I, Palmer GH, Bock R, Pedroni MJ, Rameshkumar M, Ferreira MB, Fletcher TI, McElwain TF: Attenuation of virulence in an apicomplexan hemoparasite results in reduced genome diversity at the population level. BMC Genomics 2011, 12:410.

43. Sivakumar $T$, Tagawa M, Yoshinari $T$, Ybañez AP, Igarashi I, Ikehara $Y$, Hata H, Kondo S, Matsumoto K, Inokuma H, Yokoyama N: PCR detection of Babesia ovata from cattle reared in Japan and clinical significance of coinfection with Theileria orientalis. J Clin Microbiol 2012, 50:2111-2113

44. Belongia EA: Epidemiology and impact of coinfections acquired from Ixodes ticks. Vector Borne Zoonotic Dis 2002, 2:265-273.

45. Halos L, Jamal T, Maillard R, Beugnet F, Le Menach A, Boulouis H-J, Vayssier-Taussat M: Evidence of Bartonella sp. in questing adult and nymphal Ixodes ricinus ticks from France and co-infection with Borrelia burgdorferi sensu lato and Babesia sp. Vet Res 2005, 36:79-87.

46. Milutinović M, Masuzawa T, Tomanović S, Radulović Ž, Fukui T, Okamoto Y: Borrelia burgdorferi sensu lato, Anaplasma phagocytophilum, Francisella tularensis and their co-infections in host-seeking Ixodes ricinus ticks collected in Serbia. Exp Appl Acarol 2008, 45:171-183.

47. Piccolin G, Benedetti G, Doglioni C, Lorenzato C, Mancuso S, Papa N, Pitton L, Ramon MC, Zasio C, Bertiato G: A study of the presence of $B$. burgdorferi, Anaplasma (previously Ehrlichia) phagocytophilum, Rickettsia, and Babesia in Ixodes ricinus collected within the territory of Belluno, Italy. Vector Borne Zoonotic Dis 2006, 6:24-31.

48. Purnell RE, Young ER, Brocklesby DW, Hendry DJ: The haematology of experimentally-induced $B$. divergens and E. phagocytophila infections in splenectomised calves. Vet Rec 1977, 100:4-6.

49. Alani AJ, Herbert IV: The morphometrics of Babesia motasi (Wales) and its transmission by Haemaphysalis punctata (Canestrini and Fanzago 1877) to sheep. Vet Parasitol 1988, 30:87-95.

50. Lewis D, Holman MR, Purnell RE, Young ER, Herbert IV, Bevan WJ: Investigations on Babesia motasi isolated from Wales. Res Vet Sci 1981 31:239-243.

51. Uilenberg G, Rombach MC, Perié NM, Zwart D: Blood parasites of sheep in the Netherlands. II. Babesia motasi (Sporozoa, Babesiidae). Tijdschr Diergeneeskd 1980, 105:3-14.

52. Ahmed JS, Luo J, Schnittger L, Seitzer U, Jongejan F, Yin H, Ahmed JS, Luo J, Schnittger L, Seitzer U, Jongejan F, Yin H: Phylogenetic position of small ruminant infecting piroplasms. Ann N Y Acad Sci 2006, 1081:498-504

53. Péter $\mathrm{O}$, Bretz AG, Bee D: Occurrence of different genospecies of Borrelia burgdorferi sensu lato in ixodid ticks of Valais, Switzerland. Eur J Epidemiol 1995, 11:463-467.

54. Waldrup KA, Kocan AA, Barker RW, Wagner GG: Transmission of Babesia odocoilei in white-tailed deer (Odocoileus virginianus) by Ixodes scapularis (Acari: Ixodidae). J Wildl Dis 1990, 26:390-391.

55. Øines $\varnothing$, Radzijevskaja J, Paulauskas A, Rosef O: Prevalence and diversity of Babesia spp. in questing Ixodes ricinus ticks from Norway. Parasit Vectors 2012, 5:156.
56. Gern L, Morán Cadenas F, Burri C: Influence of some climatic factors on Ixodes ricinus ticks studied along altitudinal gradients in two geographic regions in Switzerland. Int J Med Microbiol 2008, 298(Suppl 1):55-59.

57. Jouda F, Perret J-L, Gern L: Ixodes ricinus density, and distribution and prevalence of Borrelia burgdorferi sensu lato infection along an altitudinal gradient. J Med Entomol 2004, 41:162-169.

doi:10.1186/1297-9716-45-65

Cite this article as: Michel et al:: Babesia spp. in European wild ruminant species: parasite diversity and risk factors for infection. Veterinary Research 2014 45:65.

\section{Submit your next manuscript to BioMed Central and take full advantage of:}

- Convenient online submission

- Thorough peer review

- No space constraints or color figure charges

- Immediate publication on acceptance

- Inclusion in PubMed, CAS, Scopus and Google Scholar

- Research which is freely available for redistribution

Submit your manuscript at www.biomedcentral.com/submit
C Biomed Central 